\title{
IDEMPOTENT-GENERATED SEMIGROUPS AND PSEUDOVARIETIES
}

\author{
J. ALMEIDA AND A. MOURA
}

\begin{abstract}
The operator which constructs the pseudovariety generated by the idempotent-generated semigroups of a given pseudovariety is investigated. Several relevant examples of pseudovarieties generated by their idempotentgenerated elements are given as well as some properties of this operator. Particular attention is paid to the pseudovarieties in $\{\mathrm{J}, \mathrm{R}, \mathrm{L}, \mathrm{DA}\}$ concerning this operator and their generator ranks and idempotent-generator ranks.
\end{abstract}

\section{INTRODUCTION}

Due to its applications in Computer Science, the theory of finite semigroups saw significant advancements in the 1960's driven by developments in the theory of finite automata. This connection with finite semigroups was firstly explored to obtain computational results. In parallel, combinatorial and algebraic properties of finite semigroups were studied. Eilenberg [6] established a correspondence between certain families of rational languages and certain classes of finite semigroups, called pseudovarieties, which provided a suitable framework for the bridges between the two theories.

There are many important pseudovarieties, often constructed from other ones by applying suitable operators. Some natural operators have been extensively studied. In this paper, we introduce a new one which constructs the subpseudovariety generated by the idempotent-generated semigroups of a given pseudovariety.

Several works have been dedicated to idempotent-generated semigroups. It is well-known that any finite semigroup embeds into a finite regular idempotentgenerated semigroup, which was proved by Howie [8] using full transformations semigroups. Howie [9] also proved that the full transformations subsemigroup consisting of all order-preserving and contractive full transformations is idempotentgenerated. Laradji and Umar [10] improved this result and showed that, in fact, every order-preserving and contractive full transformation is expressible as a product of idempotents of the same type and with the same range. The analogous result for the subsemigroup of contractive full transformations also holds [10.

On the other hand, Pastijn [13] proved that every completely simple semigroup embeds into an idempotent-generated one. Furthermore, Petrich [14 presents a concrete model of the embedding due to Pastijn [13] of a semigroup into an idempotentgenerated Rees matrix semigroup that preserves some properties.

In this paper, while we do not obtain a complete characterization of the pseudovarieties which are generated by their idempotent-generated semigroups, we prove

2010 Mathematics Subject Classification. 20M07.

Key words and phrases. Finite semigroup; pseudovariety; operator; idempotent-generated semigroup; generator rank; idempotent generator rank. 
that many familiar pseudovarieties have this property. The techniques used for this purpose include the representations of free profinite semigroups over R, J and DA due to Almeida and Weil [3, Almeida 11, and Moura [11, respectively. In the cases of $\mathrm{R}$ and $\mathrm{J}$, we also observe an alternative approach based on some results concerning transformations of a finite chain due to Pin [15] and Straubing [19] and the results concerning idempotent-generated subsemigroups of full transformations from Howie, and Laradji and Umar. On the other hand, the work of Petrich [14] allows us to show that the pseudovarieties $\bar{H}$, where $H$ is a pseudovariety of groups, CS and CR also have this property.

The new approach in the case of the pseudovarieties $R$ and $J$ is justified as it gives a significant improvement in terms of the generator rank and idempotent generator rank. More generally, we show that both ranks are infinite for every pseudovariety in the interval [J,DS]. We also prove that every semigroup in the subpseudovariety generated by all $n$-generated members of any of the pseudovarieties J, R, L, DA divides a semigroup in the same pseudovariety generated by at most $n+1$ idempotents. We compare these results with the works of Umar [20, and Laradji and Umar [10] concerning the ranks and idempotent-ranks of the subsemigroups of all contractive full transformations, and contractive and order-preserving full transformations, respectively. We observe that, in fact, we decrease the number of idempotent generators of the idempotent-generated semigroups when we use the embeddings of the semigroups of $\mathrm{R}$ and $\mathrm{J}$ presented in this paper.

The paper is organized as follows. In Section 2 we recall some basics of the theory of pseudovarieties of semigroups and profinite semigroups and we introduce some notation concerning operators on pseudovarieties. We also present a list of the pseudovarieties and bases of pseudoidentities defining them that will be used in our study. In Section 3 we observe some properties of the operator $-\mathrm{E}$, we determine some pseudovarieties of the form $V_{E}$ and we make a short introduction of the main question addressed in the paper: what are the pseudovarieties that are generated by their idempotent-generated elements? We present in the following sections some pseudovarieties having this property: in Section 4 using the embedding in a Rees matrix semigroup constructed by Petrich, and in Section [5] using representations of the free profinite semigroup. Finally, in Section [6 we determine the generator rank and idempotent generator rank of every pseudovariety in the interval [J, DS] and we also determine a lower bound for the idempotent generator rank of the subpseudovarieties generated by all $n$-generated members of any pseudovariety in the interval [J, DA]. Combining with the results of Section 5 we improve the last result showing that the lower bound is the exact value in the case of the pseudovarieties J, R, L, DA. To introduce some relevant results in our study, we develop some existing techniques that need to be recalled. Rather than including them in Section 2 we briefly introduce them when needed.

\section{Preliminaries}

We assume acquaintance with notions concerning pseudovarieties of semigroups and profinite semigroups. We briefly recall some basics and we refer the reader to [1, 2, 15, for detailed information.

For a semigroup $S$, let $S^{1}$ be the monoid obtained by adjoining a neutral element 1 to $S$ in case $S$ does not already possess one, and $S^{1}=S$ otherwise. We denote by $E(S)$ the set of idempotents of $S$ and by $\langle E(S)\rangle$ the subsemigroup of $S$ 
generated by $E(S)$. For $s \in S, s^{\omega}$ denotes the unique idempotent in the subsemigroup generated by $s$. We say that a semigroup $S$ divides a semigroup $T$, and we write $S \prec T$, if there exists a surjective homomorphism of a subsemigroup of $T$ onto $S$.

A pseudovariety of semigroups is a class of finite semigroups that is closed under taking subsemigroups, homomorphic images and finite direct products. Equivalently, a pseudovariety of semigroups is a class of finite semigroups closed under taking division and finite direct products. For example, $\mathrm{S}$ is the pseudovariety of all finite semigroups.

There are many ways to construct new pseudovarieties from known ones, that is by applying operators to pseudovarieties. For example, given a pseudovariety $\mathrm{V}$, the following classes of finite semigroups are pseudovarieties:

- EV consists of all $S \in \mathrm{S}$ such that $\langle E(S)\rangle \in \mathrm{V}$;

- DV consists of all $S \in \mathrm{S}$ such that, for every regular $\mathcal{D}$-class $D$ of $S, D \in \mathrm{V}$;

- for a pseudovariety $\mathrm{H}$ of groups, $\overline{\mathrm{H}}$ consists of all $S \in \mathrm{S}$ such that every subgroup $G$ of $S$ belongs to $\mathrm{H}$.

We also have other types of operators that construct new pseudovarieties by describing the generators. The new pseudovariety is then the smallest pseudovariety containing such semigroups. In this way, we introduce the operator $V_{E}$, which is the topic of this paper. Given a pseudovariety $V$, we define $V_{E}$ as the pseudovariety generated by the subsemigroups generated by the idempotents of each member of $\mathrm{V}$, i.e.,

$$
\mathrm{V}_{\mathrm{E}}=\langle\langle E(S)\rangle \mid S \in \mathrm{V}\rangle .
$$

Note that $\mathrm{V}_{\mathrm{E}} \subseteq \mathrm{V}$ as the indicated generators of $\mathrm{V}_{\mathrm{E}}$ belong to $\mathrm{V}$.

Because it will be useful in our study, we present an obvious observation about the subsemigroup generated by a subset of idempotents of a given semigroup:

Lemma 2.1. Let $S \in \mathrm{S}$ and $X \subseteq E(S)$. Then $\langle E\langle X\rangle\rangle=\langle X\rangle$. In particular, we have $\langle E\langle E(S)\rangle\rangle=\langle E(S)\rangle$ and $\langle E\langle E(D)\rangle\rangle=\langle E(D)\rangle$, for every regular $\mathcal{D}$-class $D$ of $S$.

A semigroup equipped with a topology for which the multiplication is a continuous function is called a topological semigroup. Finite semigroups are endowed with the discrete topology. A topological semigroup $S$ is a profinite semigroup (respectively, a pro-V semigroup) if it is a compact semigroup which is residually finite (respectively, residually in $\mathrm{V}$ ), which means that, for any two distinct elements of $S$, there exists a continuous homomorphism into a finite semigroup (respectively, into a member of $\mathrm{V}$ ) that separates them. Equivalently, profinite semigroups are compact 0-dimensional, which means that the topology has an open basis consisting of clopen sets. The elements of a pseudovariety $\mathrm{V}$ are pro- $\mathrm{V}$ semigroups.

We denote by $\bar{\Omega}_{A} \vee$ the free pro- $\mathrm{V}$ semigroup on $A$, which is the unique (up to isomorphism of topological semigroups) pro- $\mathrm{V}$ semigroup such that every mapping $\mu: A \rightarrow S$ into a pro-V semigroup $S$ can be extended to a unique continuous homomorphism $\hat{\mu}: \bar{\Omega}_{A} \mathrm{~V} \rightarrow S$ such that $\hat{\mu} \circ \iota=\mu$, where $\iota: A \rightarrow \bar{\Omega}_{A} \mathrm{~V}$ is the natural generating function (i.e., its image generates a dense subsemigroup of $\bar{\Omega}_{A} \mathrm{~V}$ ). The elements of $\bar{\Omega}_{A} \mathrm{~V}$ are called implicit operations over $\mathrm{V}$. For $u \in \bar{\Omega}_{A} \mathrm{~V}$ the sequence $\left(u^{n !}\right)_{n}$ converges and we denote the limit by $u^{\omega}$, which is the unique idempotent in the closed subsemigroup generated by $u$. 
An equality of the form $u=v$, with $u, v \in \bar{\Omega}_{A} \mathrm{~S}$, is called a pseudoidentity and $|A|$ is its arity. The pseudoidentity is valid in a profinite semigroup $S$, and we write $S \models u=v$, if, for every continuous homomorphism $\varphi: \bar{\Omega}_{A} \mathrm{~S} \rightarrow S$, we have $\varphi(u)=\varphi(v)$. It is easy to see that the validity of a pseudoidentity in a finite semigroup is preserved under taking homomorphic images, subsemigroups and finite direct products. Conversely, Reiterman's Theorem [17 says that every pseudovariety is defined by some set of finitary pseudoidentities. We end this section with a list of pseudovarieties that will be used in this paper and some corresponding bases of pseudoidentities defining them:

$$
\begin{aligned}
\mathrm{J} & =\llbracket(x y)^{\omega} x=(x y)^{\omega}=y(x y)^{\omega} \rrbracket & & \mathcal{J} \text {-trivial semigroups; } \\
\mathrm{R} & =\llbracket(x y)^{\omega} x=(x y)^{\omega} \rrbracket & & \mathcal{R} \text {-trivial semigroups; } \\
\mathrm{L} & =\llbracket y(x y)^{\omega}=(x y)^{\omega} \rrbracket & & \mathcal{L} \text {-trivial semigroups; } \\
\mathrm{A} & =\llbracket x^{\omega+1}=x^{\omega} \rrbracket & & \text { aperiodic (or } \mathcal{H} \text {-trivial) semigroups; } \\
\mathrm{G} & =\llbracket x^{\omega}=1 \rrbracket & & \text { groups; } \\
\mathrm{LG} & =\llbracket\left(x^{\omega} y\right)^{\omega} x^{\omega}=x^{\omega} \rrbracket & & \text { local groups; } \\
\mathrm{CR} & =\llbracket x^{\omega+1}=x \rrbracket & & \text { completely regular semigroups; } \\
\mathrm{CS} & =\llbracket x^{\omega+1}=x,(x y x)^{\omega}=x^{\omega} \rrbracket & & \text { completely simple semigroups; } \\
\mathrm{RB} & =\llbracket x^{2}=x, x y x=x \rrbracket & & \text { rectangular bands; } \\
\mathrm{DA} & =\llbracket\left((x y)^{\omega} x\right)^{2}=(x y)^{\omega} x \rrbracket & & \text { regular } \mathcal{D} \text {-classes are aperiodic semigroups; } \\
\mathrm{DG} & =\llbracket(x y)^{\omega}=(y x)^{\omega} \rrbracket & & \text { regular } \mathcal{D} \text {-classes are groups; } \\
\mathrm{DO} & =\llbracket(x y)^{\omega}(y x)^{\omega}(x y)^{\omega}=(x y)^{\omega} \rrbracket & & \text { regular } \mathcal{D} \text {-classes are orthodox semigroups; } \\
\mathrm{DS} & =\llbracket\left((x y)^{\omega} x\right)^{\omega+1}=(x y)^{\omega} x \rrbracket & & \text { regular } \mathcal{D} \text {-classes are semigroups. }
\end{aligned}
$$

We reserve the letter $\mathrm{H}$ to denote an arbitrary pseudovariety of groups.

\section{Properties of the operator -E}

We establish some basic properties of the operator $-E$. We start by observing that the definition given for $V_{E}$, where $V$ is a pseudovariety, is equivalent to $V_{E}$ being generated by the idempotent-generated semigroups of $\mathrm{V}$.

Lemma [3.1] and its corollaries below can be extracted from [4] where the operators $-E$ and $E_{\text {_ }}$ are studied from the viewpoint of congruences on the lattice of pseudovarieties of finite semigroups. The proofs are presented here for the sake of completeness.

Lemma 3.1. The operator $-\mathrm{E}$ has the following properties, where $\mathrm{V}$ and $\mathrm{W}$ are arbitrary pseudovarieties:

(1) $\mathrm{V}_{\mathrm{E}}=\langle T \in \mathrm{V} \mid T=\langle E(T)\rangle\rangle$;

(2) $\mathrm{V} \subseteq \mathrm{W}$ implies $\mathrm{V}_{\mathrm{E}} \subseteq \mathrm{W}_{\mathrm{E}}$;

(3) $(\mathrm{V} \cap \mathrm{W})_{\mathrm{E}} \subseteq \mathrm{V}_{\mathrm{E}} \cap \mathrm{W}_{\mathrm{E}}$;

(4) $\left(\mathrm{V}_{\mathrm{E}}\right)_{\mathrm{E}}=\mathrm{V}_{\mathrm{E}}$;

(5) $(\mathrm{EV})_{\mathrm{E}}=\mathrm{V}_{\mathrm{E}}$;

(6) $\mathrm{E}\left(\mathrm{V}_{\mathrm{E}}\right)=\mathrm{EV}$. 
Proof. (11) Let $T=\langle E(S)\rangle$ with $S \in \mathrm{V}$. Since $\langle E(S)\rangle$ is a subsemigroup of $S$, it follows that $T \in \mathrm{V}$. By Lemma 2.1 we have that $\langle E(T)\rangle=\langle E\langle E(S)\rangle\rangle=\langle E(S)\rangle=$ $T$. Hence the generators of the two pseudovarieties are the same.

(2) is immediate from the definition of $V_{E}$ and (3) follows from (2).

(41) The direct inclusion follows from $\mathrm{V}_{\mathrm{E}} \subseteq \mathrm{V}$ and (2). Conversely, since the generators of $\mathrm{V}_{\mathrm{E}}$ are the semigroups $\langle E(S)\rangle$, with $S \in \mathrm{V}$, it suffices to show that $\langle E(S)\rangle \in\left(\mathrm{V}_{\mathrm{E}}\right)_{\mathrm{E}}$, for all $S \in \mathrm{V}$. Indeed, since $\langle E(S)\rangle \in \mathrm{V}_{\mathrm{E}}$, by Lemma 2.1 and definition of ${ }_{-\mathrm{E}}$ we have $\langle E(S)\rangle=\langle E\langle E(S)\rangle\rangle \in\left(\mathrm{V}_{\mathrm{E}}\right)_{\mathrm{E}}$.

(15) We have $(\mathrm{EV})_{\mathrm{E}}=\langle\langle E(S)\rangle \mid S \in \mathrm{EV}\rangle=\langle\langle E(S)\rangle \mid\langle E(S)\rangle \in \mathrm{V}\rangle$. Let us see that the generators of $(\mathrm{EV})_{\mathrm{E}}$ are in $\mathrm{V}_{\mathrm{E}}$. In fact, as $\langle E(S)\rangle \in \mathrm{V}$, Lemma 2.1 yields $\langle E(S)\rangle=\langle E\langle E(S)\rangle\rangle \in \mathrm{V}_{\mathrm{E}}$. The reverse inclusion follows from $\mathrm{V} \subseteq \mathrm{EV}$ and (2).

(6) Since $\mathrm{V}_{\mathrm{E}} \subseteq \mathrm{V}$, applying the increasing operator $\mathrm{E}_{-}$, we obtain $\mathrm{E}\left(\mathrm{V}_{\mathrm{E}}\right) \subseteq \mathrm{EV}$. If $S \in \mathrm{EV}$, i.e., $\langle E(S)\rangle \in \mathrm{V}$, then Lemma 2.1 gives that $\langle E(S)\rangle=\langle E\langle E(S)\rangle\rangle$ is one of the generators of $\mathrm{V}_{\mathrm{E}}$ so that, in particular, $S \in \mathrm{E}\left(\mathrm{V}_{\mathrm{E}}\right)$.

A natural question, for which we have no answer, is whether we always have equality in part (3) of Lemma 3.1. More generally, one may ask whether the operator -E preserves arbitrary intersections, while it is easy to see that it preserves arbitrary joins in the lattice of all pseudovarieties of finite semigroups. The more general question can be viewed as one of many similar questions arising from [4. See 16 for related questions.

Corollary 3.2. Let $\mathrm{V}$ and $\mathrm{W}$ be pseudovarieties such that $\mathrm{V}_{\mathrm{E}}=\mathrm{V}$ and $\mathrm{EV}=\mathrm{EW}$. Then $\mathrm{V} \subseteq \mathrm{W}$.

Proof. Applying the operator $-\mathrm{E}$ to $\mathrm{EV}=\mathrm{EW}$ and using property (5) of Lemma 3.1. it follows that $\mathrm{V}=\mathrm{V}_{\mathrm{E}}=(\mathrm{EV})_{\mathrm{E}}=(\mathrm{EW})_{\mathrm{E}}=\mathrm{W}_{\mathrm{E}} \subseteq \mathrm{W}$

Corollary 3.3. Given two pseudovarieties $\mathrm{V}$ and $\mathrm{W}$, the following conditions are equivalent:

(a) $\mathrm{V}_{\mathrm{E}}=\mathrm{W}_{\mathrm{E}}$;

(b) $\mathrm{EV}=\mathrm{EW}$;

(c) $\mathrm{V}_{\mathrm{E}} \subseteq \mathrm{W} \subseteq \mathrm{EV}$

Proof. (a) $\Rightarrow$ (b) From (a) and property (6) of Lemma 3.1] it follows that EV = $\mathrm{E}\left(\mathrm{V}_{\mathrm{E}}\right)=\mathrm{E}\left(\mathrm{W}_{\mathrm{E}}\right)=\mathrm{EW}$.

(b) $\Rightarrow$ (C) The second inclusion in (C) follows from $W \subseteq E W=E V$. To show the first inclusion, we recall that, by properties (4)-(6) of Lemma 3.1 $\left((E V)_{\mathrm{E}}\right)_{\mathrm{E}}=(\mathrm{EV})_{\mathrm{E}}$ and $\mathrm{E}\left((\mathrm{EV})_{\mathrm{E}}\right)=\mathrm{EV}=\mathrm{EW}$. Hence, by Corollary $3.2(\mathrm{EV})_{\mathrm{E}} \subseteq \mathrm{W}$. Moreover, also by property (5) of Lemma $3.1 \mathrm{~V}_{\mathrm{E}}=(\mathrm{EV})_{\mathrm{E}} \subseteq \mathrm{W}$.

$($ C $) \Rightarrow($ al $)$ Applying the operator -E to (C) , by properties (2), (4) and (5) of Lemma 3.1 we obtain $\mathrm{V}_{\mathrm{E}}=\left(\mathrm{V}_{\mathrm{E}}\right)_{\mathrm{E}} \subseteq \mathrm{W}_{\mathrm{E}} \subseteq(\mathrm{EV})_{\mathrm{E}}=\mathrm{V}_{\mathrm{E}}$ and, therefore, $\mathrm{W}_{\mathrm{E}}=$ $\mathrm{V}_{\mathrm{E}}$.

In other words, given a pseudovariety $\mathrm{V}$, the equations $\mathrm{X}_{\mathrm{E}}=\mathrm{V}_{\mathrm{E}}$ and $\mathrm{EX}=\mathrm{EV}$ in the variable $X$ are equivalent and the class of its solutions is the interval [ $\left.V_{E}, E V\right]$.

It is natural to ask for which pseudovarieties $V, V_{E}$ is equal to $V$. As an obvious example, for every pseudovariety $\mathrm{V}$ of bands, since its semigroups consist only of idempotents, we have $\mathrm{V}_{\mathrm{E}}=\mathrm{V}$. But, there are pseudovarieties that do not satisfy the equality $\mathrm{V}=\mathrm{V}_{\mathrm{E}}$. Let us see some examples: 
Example 3.4. For every pseudovariety $\mathrm{H}$ of groups, we have $\mathrm{H}_{\mathrm{E}}=\mathrm{I}$, where $\mathrm{I}=$ $\llbracket x=y \rrbracket$ is the trivial pseudovariety.

Example 3.5. It is well known that LG is the class of all finite semigroups such that all idempotents are $\mathcal{J}$-equivalent and, therefore, they are in the minimal ideal of the semigroup. So we have $(\mathrm{LG})_{\mathrm{E}}=\langle\langle E(S)\rangle \mid S \in \mathrm{LG}\rangle \subseteq \mathrm{CS} \varsubsetneqq \mathrm{LG}$.

Example 3.6. It is well known that $\mathrm{RB} \vee \mathrm{G}=\mathrm{CS} \cap \mathrm{O}$, where $\mathrm{O}=\llbracket\left(x^{\omega} y^{\omega}\right)^{\omega}=x^{\omega} y^{\omega} \rrbracket$ is the class of all finite orthodox semigroups. So we have $(R B \vee G)_{E}=(C S \cap O)_{E}=$ RB.

The notion of E-local pseudovariety, introduced in 12, enables us to determine $(\mathrm{DO})_{\mathrm{E}}$ and $(\mathrm{DH})_{\mathrm{E}}$, as we see in the following examples. Recall that a pseudovariety $\mathrm{V}$ is E-local if it satisfies the following property: given $S \in \mathrm{S},\langle E(S)\rangle \in \mathrm{V}$ if and only if $\langle E(D)\rangle \in \mathrm{V}$, for every regular $\mathcal{D}$-class $D$ of $S$.

Example 3.7. Let $S \in \mathrm{DH}$. Since every regular $\mathcal{D}$-class $D$ of $S$ is a group, it follows that $\langle E(D)\rangle$ is trivial and, therefore, $\langle E(D)\rangle \in \mathrm{J}$. Since J is E-local (see 12 , Example 3.6]), we have $\langle E(S)\rangle \in \mathrm{J}$. Hence $\mathrm{J} \subseteq \mathrm{DH} \subseteq \mathrm{EJ}$, where the first inclusion is trivial. Thus it follows from Corollary 3.3 that $(\mathrm{DH})_{\mathrm{E}}=J_{\mathrm{E}}=\mathrm{J}$, where the last equality follows from Corollary 3.14 which is proved below.

Example 3.8. We observe that DO $\subseteq$ EDA. Indeed, for $S \in \mathrm{DO}$ and a regular $\mathcal{D}$-class $D$ of $S,\langle E(D)\rangle \in \mathrm{DA}$. Since DA is E-local (see [12 Proposition 3.5]), we have $\langle E(S)\rangle \in \mathrm{DA}$. Hence $S \in \mathrm{EDA}$. Since DA $\subseteq \mathrm{DO} \subseteq \mathrm{EDA}$, it follows from Corollary 3.3 that $(\mathrm{DO})_{\mathrm{E}}=(\mathrm{DA})_{\mathrm{E}}=\mathrm{DA}$, where the last equality follows from Corollary [5.6 which is established in Section $[5$

In an attempt to identify the pseudovarieties which are generated by their idempotent-generated elements, we present some results in the following sections. We start by suggesting, as an easy exercise, the result from Howie [8] which states that any finite semigroup embeds into a finite regular idempotent-generated semigroup, so that, in particular, we have the following result.

Proposition 3.9 (cf. Howie 8 ) $) \mathrm{S}_{\mathrm{E}}=\mathrm{S}$.

On the other hand, Pin [15] and Straubing [19] obtained the following representation theorems for $\mathcal{R}$-trivial monoids and $\mathcal{J}$-trivial monoids, respectively.

Theorem 3.10 (cf. Pin [15, Theorem IV.3.6]). A finite monoid is $\mathcal{R}$-trivial if and only if it is a submonoid of $\mathcal{E}_{X}$, the submonoid consisting of all contractive total transformations of some finite chain $X$.

Theorem 3.11 (cf. Straubing [19]). A finite monoid is $\mathcal{J}$-trivial if and only if it divides $\mathcal{C}_{X}$, the submonoid of all order-preserving and contractive transformations of some finite chain $X$.

Combining these theorems with the following results about idempotent-generated subsemigroups of total transformations due, respectively, to Laradji and Umar [10] and to Howie 9, we obtain Corollary 3.14

Theorem 3.12 (cf. Laradji and Umar [10, Theorem 1.3]). The monoid $\mathcal{E}_{X}$ is idempotent-generated.

Theorem 3.13 (cf. Howie 9, Theorem 3.2]). The monoid $\mathcal{C}_{X}$ is idempotentgenerated. 
Corollary 3.14. The equality $\mathrm{V}_{\mathrm{E}}=\mathrm{V}$ holds if $\mathrm{V}$ is any of the pseudovarieties $R, L, J$.

Similarly, using embeddings into idempotent-generated semigroups of the same type from Pastijn [13] concerning completely simple semigroups and completely regular semigroups, we obtain the following results.

Proposition 3.15 (cf. Pastijn [13, Corollary 3.7]). $(\mathrm{CS})_{\mathrm{E}}=\mathrm{CS}$.

Proposition 3.16 (cf. Pastijn [13, Theorem 3.5]) $(\mathrm{CR})_{\mathrm{E}}=\mathrm{CR}$.

Using Proposition [3.15] we may establish an equality in Example 3.5] as we see below.

Example 3.17. By Proposition 3.15 property (2) of Lemma 3.1 and since CS $\subseteq$ $\mathrm{LG}$, it follows that $\mathrm{CS}=(\mathrm{CS})_{\mathrm{E}} \subseteq(\mathrm{LG})_{\mathrm{E}}$. Thus, and by Example 3.5. we have $(\mathrm{LG})_{\mathrm{E}}=\mathrm{CS}$.

In Section 4 we return to these last two results and we show how to prove them using the general construction of Petrich [14. In Section 5 we prove that the pseudovarieties R, L and J are fixed points of the -E operator by a different approach, namely by using implicit operations. While using transformation semigroups the number of idempotent generators of the idempotent-generated semigroup depends on the cardinality of the embedded semigroup, in this method the number of idempotent generators of the idempotent-generated semigroup is controlled by the number of generators of the embedded semigroup. However, for the case $V=R$, the first method enables us to show that, in fact, there exists an embedding of an $\mathcal{R}$ trivial semigroup into an idempotent-generated $\mathcal{R}$-trivial semigroup. In the other cases, we just prove a division property. The second method is also used to prove the equality for the pseudovariety DA, while we do not know how to apply the first method.

\section{An embedding into a Rees matrix semigroup}

In 5] one can find a proof of Howie's result that every (finite) semigroup can be embedded into an idempotent-generated (finite) semigroup. The proof is attributed to J.-F. Perrot and depends on a construction that was later used and extended by Pastijn 13 to obtain such embedding results within special classes of semigroups. Petrich 14 reformulated Perrot's construction in a new notation that made it considerably more transparent. We reformulate Pastijn's embedding results for completely simple [13, Corollary 3.7] and completely regular [13, Theorem 3.5] semigroups in Petrich's language.

We briefly recall the construction of this embedding. Let $S$ be a semigroup (not necessarily finite). We consider the Rees matrix semigroup $\Phi S=\mathcal{M}\left(S^{1}, S^{1}, \Sigma ; Q\right)$, with $\Sigma=\{\sigma, \tau\}$, where $\sigma$ and $\tau$ are two distinct symbols that are not in $S$, and $Q=\left(q_{\alpha s}\right)$ is the sandwich matrix with entries

$$
q_{\sigma s}=1, \quad q_{\tau s}=s \quad\left(s \in S^{1}\right) .
$$

The mapping

$$
\varphi_{S}: s \rightarrow(1, s, \sigma) \quad(s \in S)
$$

embeds $S$ into $\Phi S$, although it is not the unique embedding from $S$ into $\Phi S$. Petrich determined the set of idempotents of $\Phi S$, which is

$$
E(\Phi S)=\left\{(s, t, \sigma) \in \Phi S \mid t \in E\left(S^{1}\right)\right\} \cup\{(s, t, \tau) \in \Phi S \mid t=t s t\}
$$


and described Green's relations on $\Phi S$ as follows.

Lemma 4.1 (Petrich [14, Lemma 4.3]). Let $(s, t, \alpha),(u, v, \beta) \in \Phi S$. Then:

(1) $(s, t, \alpha) \mathcal{L}(u, v, \beta)$ if and only if $t \mathcal{L} v$ and $\alpha=\beta$;

(2) $(s, t, \alpha) \mathcal{R}(u, v, \beta)$ if and only if $s=u$ and $t \mathcal{R} v$;

(3) $(s, t, \alpha) \mathcal{H}(u, v, \beta)$ if and only if $s=u, t \mathcal{H} v$, and $\alpha=\beta$;

(4) $(s, t, \alpha) \mathcal{D}(u, v, \beta)$ if and only if $t \mathcal{D} v$.

Thus, $\Phi S$ has the same number of $\mathcal{D}$-classes as $S^{1}$ and each $\mathcal{D}$-class $D^{\prime}$ of $\Phi S$, which corresponds to a $\mathcal{D}$-class $D$ of $S$, has the following number respectively of $\mathcal{L}$-classes and $\mathcal{R}$-classes: $2 \cdot \mid \mathcal{L}$-classes of $D \mid$ and $\left|S^{1}\right| \cdot \mid \mathcal{R}$-classes of $D \mid$. Each $\mathcal{H}$-class of $\Phi S$ has the same number of elements of the corresponding $\mathcal{H}$-class in $S^{1}$.

It is obvious that if $S$ is a finite semigroup, so is $\Phi S$. Note that $\Phi S$ is generated by the set of idempotents $\left\{(s, 1, \sigma) \mid s \in S^{1}\right\} \cup\{(1,1, \tau)\}$, which gives an immediate proof of the result from Howie (see Proposition [3.9). Petrich also showed that this embedding preserves other properties of $S$ (see [14 Theorem 5.4]). In particular, he proves the following corollary.

Corollary 4.2 (cf. Petrich [14, Theorem 5.4]). Every semigroup of $\overline{\mathrm{H}}$, where $\mathrm{H}$ is a pseudovariety of groups, embeds into an idempotent-generated semigroup of $\overline{\mathrm{H}}$.

Choosing some specific subsemigroups of $\Phi S$, we can prove the following results:

Proposition 4.3. Every semigroup of CS embeds into an idempotent-generated semigroup of $\mathrm{CS}$.

Proof. Note that, if $S \in \mathrm{CS}$, then $\Phi S$ has at most two $\mathcal{D}$-classes, the one corresponding to $D, D^{\prime}$, and the other corresponding to the neutral element added to $S$. If we show that $D^{\prime}$ is generated by its idempotents, then it suffices to consider the embedding $\varphi_{S}^{\prime}: s \mapsto(1, s, \sigma)$ from $S$ into the semigroup $D^{\prime}$.

Let $(1, s, \sigma)$, with $s \in S$, be any element of $\varphi_{S}(S)$ and let $e \in E(S)$ be such that $e \mathcal{H} s$. Then one can compute $(1, e, \tau) \cdot(s, e, \sigma)=(1, s, \sigma)$ with $(1, e, \tau),(s, e, \sigma) \in$ $E\left(D^{\prime}\right)$. Hence, the group $\mathcal{H}$-class $\{(1, s, \sigma) \mid s \in S\}$ is contained in $\left\langle E\left(D^{\prime}\right)\right\rangle$. Since all $\mathcal{H}$-classes of $\left\langle E\left(D^{\prime}\right)\right\rangle$ have the same number of elements, we conclude that $\left\langle E\left(D^{\prime}\right)\right\rangle=D^{\prime}$. Hence $S$ embeds into $\left\langle E\left(D^{\prime}\right)\right\rangle$, which is an idempotent-generated completely simple semigroup.

Proposition 4.4. Every semigroup of CR embeds into an idempotent-generated semigroup of $\mathrm{CR}$.

Proof. Let $S \in \mathrm{CR}$. We want to determine an idempotent-generated completely regular subsemigroup of $\Phi S$ where $S$ embeds. Let $H$ be an $\mathcal{H}$-class of $S$. Since $H$ is a group, then the $\mathcal{H}$-classes of $\Phi S$ of the form $\{s\} \times H \times\{\sigma\}$, with $s \in S^{1}$, and $\{1\} \times H \times\{\tau\}$ are groups. Let $t$ be any element of $S$ and $H_{t}$ be the $\mathcal{H}$-class of $S$ containing this element. We observe that the $\mathcal{H}$-classes of the form $\{s\} \times H_{t} \times\{\tau\}$, with $s \geq_{\mathcal{J}} t$, are groups. Let $e \in E(S)$ be such that $e \mathcal{H} t$. Then there exist $x, y \in S$ such that $e=x s y=$ exsye. Hence $e \mathcal{L}$ sye and, since $L_{e} \cap R_{\text {sye }}$ is a group, we have that esye $\mathcal{H}$ e and es $\mathcal{R}$ e. By Green's Lemma, it follows that

$$
\begin{aligned}
\mu_{s}: H_{t} & \rightarrow H_{t s} \\
u & \mapsto u s
\end{aligned}
$$


is a bijection. Let $v \in H_{t}$ be such that $v s$ is the idempotent of $H_{t s}$. Since $v s v \mathcal{H} v$ and $\mu_{s}(v s v)=v s v s=v s=\mu_{s}(v)$, then $v s v=v$ and $(s, v, \tau)$ is the idempotent of $\{s\} \times H_{t} \times\{\tau\}$.

Now, we observe that if an $\mathcal{H}$-class of $\Phi S$ of the form $\{s\} \times H_{t} \times\{\tau\}$ is a group, then $s \geq_{\mathcal{J}} t$. Thus, if there exists $u \in H_{t}$ such that $(s, u, \tau)$ is an idempotent, then $u s u=u$, and so $s \geq \mathcal{J} u \mathcal{H} t$. So, we have identified all maximal subgroups of $\Phi S$.

We consider the subsemigroup $T$ of $\Phi S$ generated by the following idempotents:

$$
\begin{aligned}
& (s, t, \sigma) \text { with } t \in E\left(S^{1}\right) \text { and } s \geq \mathcal{J} t ; \\
& (s, t, \tau) \text { with } t=t s t .
\end{aligned}
$$

Basically, we chose all idempotents of the $\mathcal{R}$-classes whose $\mathcal{H}$-classes are groups. Thus, the product of any two idempotents on a same $\mathcal{D}$-class is also on this $\mathcal{D}$-class and, specifically, on a $\mathcal{H}$-class containing an idempotent of the set of generators. Let us see what is the product of two idempotents of the set of generators that are not in the same $\mathcal{D}$-class. Let $(s, t, \alpha)$ and $(u, v, \beta)$ be two such idempotents. We have $(s, t, \alpha) \cdot(u, v, \beta)=\left(s, t q_{\alpha u} v, \beta\right)$. As $s \geq_{\mathcal{J}} t$, then $s \geq_{\mathcal{J}} t q_{\alpha u} v$. Hence this product is in an $\mathcal{H}$-class that contains an idempotent of the set of generators of $T$.

Note that the $\mathcal{H}$-classes of the form $\{1\} \times H \times\{\sigma\}$, where $H$ is an $\mathcal{H}$-class of $S^{1}$ are in $T$. In fact, given $a \in S^{1}$, we have $(1, a, \sigma)=(1, e, \tau) \cdot(a, e, \sigma)$, where $e \in E\left(S^{1}\right)$ is such that $e \mathcal{H} a$, and $(1, e, \tau)$ and $(a, e, \sigma)$ are idempotents of $T$. It follows that $T$ is the subsemigroup of $\Phi S$ consisting of the $\mathcal{R}$-classes of $\Phi S$ whose $\mathcal{H}$-classes are groups.

Hence $T$ is a completely regular semigroup and $\varphi_{S}^{\prime \prime}: s \mapsto(1, s, \sigma)$ is an embedding of $S$ into $T$.

In the above proof, we may reduce the choice of the idempotents and we may consider the subsemigroup of $T$ generated by the following idempotents:

$$
(1, e, \tau),(a, e, \sigma) \text { with } a \in S \text { and } e \in E(S) \text { such that } e \mathcal{H} a \text {. }
$$

This subsemigroup is also a union of $\mathcal{R}$-classes of $\Phi S$ whose $\mathcal{H}$-classes are groups and the $\mathcal{H}$-classes of the form $\{1\} \times H \times\{\sigma\}$, where $H$ is an $\mathcal{H}$-class of $S^{1}$, are also in this subsemigroup. However, to simplify the proof, we considered the subsemigroup $T$.

Example 4.5. Consider the completely regular semigroup

$$
\begin{gathered}
S=\langle a, b, c, d| a^{3}=a, b^{2}=b, c^{3}=c, d^{2}=d, a b=b a, c b=b c, a d a=a, \\
a c=c a=b d=d b=c d=d c=0\rangle .
\end{gathered}
$$

We present in Figure 1 a $\mathcal{D}$-class of $\Phi S$ to illustrate the distribution of the idempotents. We also observe that $T$ consists of the $\mathcal{R}$-classes of $\Phi S$ whose $\mathcal{H}$-classes are groups, as we have mentioned previously.

Corollary 4.6. The pseudovarieties $\overline{\mathrm{H}}$, CS and $\mathrm{CR}$ satisfy the equality $\mathrm{V}_{\mathrm{E}}=\mathrm{V}$.

When we work with the pseudovarieties DS and DA, and since the regular $\mathcal{D}$ classes of the semigroups of these pseudovarieties are completely simple semigroups, one may ask whether the construction used in Proposition 4.4 may lead to a proof of existence of an embedding from every semigroup of any of these pseudovarieties into an idempotent-generated semigroup of the same pseudovariety. However, in the following example, we observe that this is not the case. 

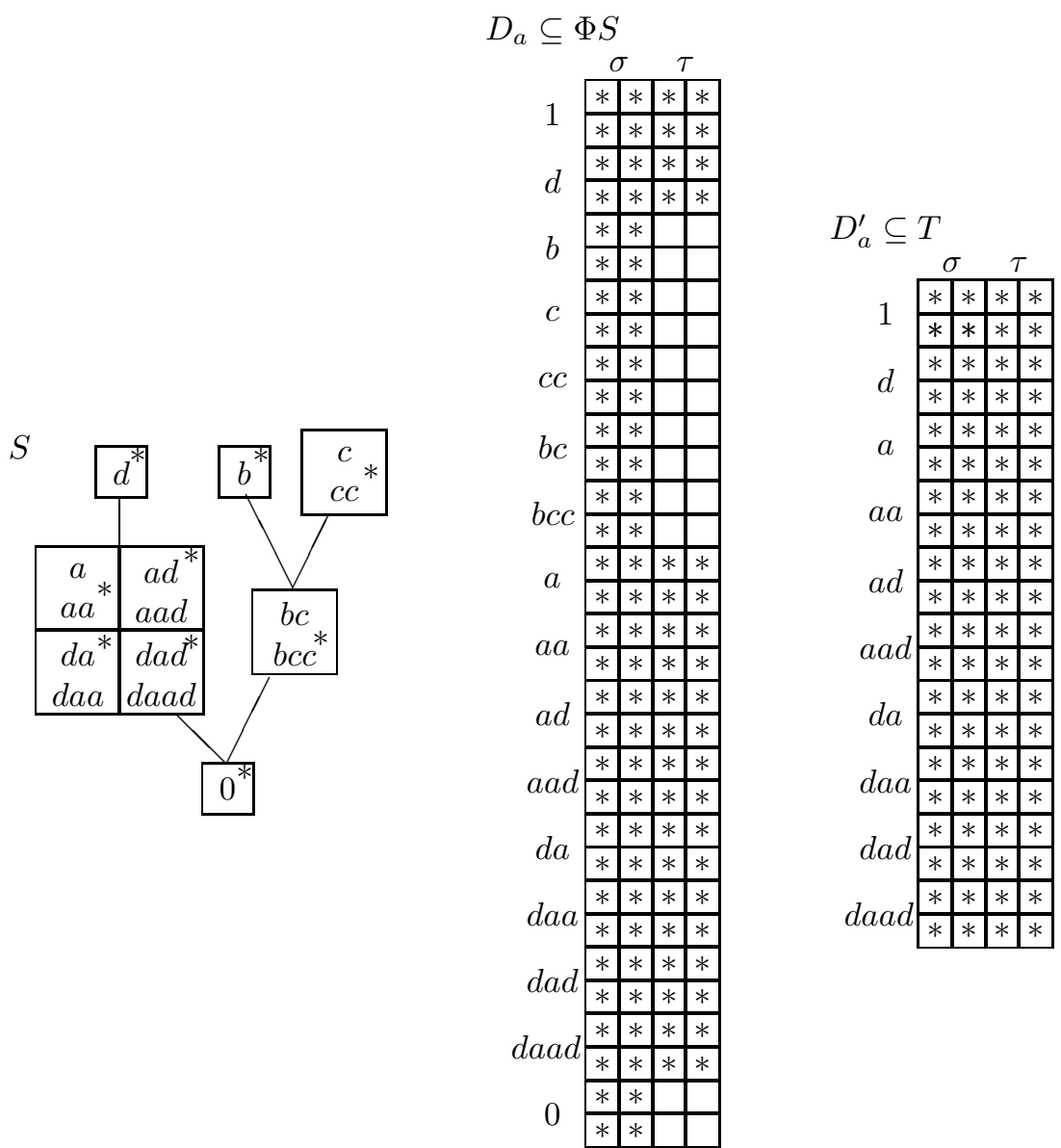

FiguRE 1. An embedding for a completely regular semigroup

Example 4.7. Consider the semigroup $S=\left\langle a \mid a^{3}=a^{2}\right\rangle$. We look at the subsemigroup $T$ of $\Phi S$ generated by the idempotents of the same type as those of Proposition 4.4 (see Figure 22). Note that neither the element $(1, a, \sigma)$ nor any element of the $\mathcal{D}$-class $D_{a}$ is in $T$. We have to choose the idempotent $(a, 1, \sigma)$ to be a generator of $T$, but, in that case, $T$ is no longer an element of DS (and, consequently, of DA).

We end this subsection with no answer for the question: Does $\mathrm{V}_{\mathrm{E}}=\mathrm{V}$ for any of the pseudovarieties DS or DA? In the following section we see that, in fact, the pseudovariety DA satisfies such equality.

\section{Representations By implicit OPERATions}

We refer the reader to 22 for detailed information about profinite semigroups and to standard references for the basics of topology. By an embedding of topological semigroups we mean a semigroup homomorphism that is simultaneously a 


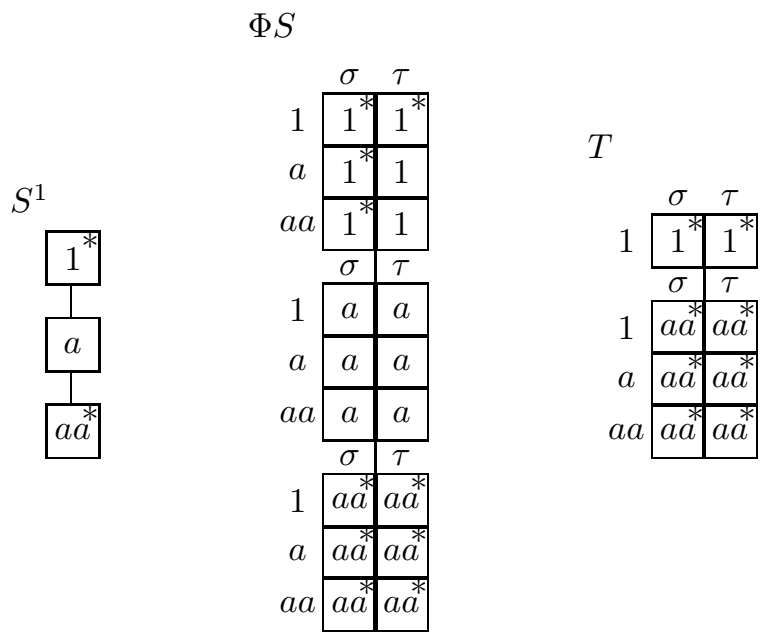

FiguRE 2. An embedding for a monogenic monoid

homeomorphism with the image subspace. A clopen subset of a topological space is one that is simultaneously closed and open.

Theorem 5.1. Let $\vee$ be a pseudovariety such that, for every $n$, there exists $m$ such that $\bar{\Omega}_{n} \mathrm{\vee}$ embeds in $\overline{\langle X\rangle}$ for some $X \subseteq E\left(\bar{\Omega}_{m} \mathrm{\vee}\right)$. Then $\mathrm{V}_{\mathrm{E}}=\mathrm{V}$.

Proof. Let $\mathrm{V}$ be a pseudovariety satisfying the above conditions. Let $S \in \mathrm{V}$ and $\varphi: \bar{\Omega}_{n} \vee \longrightarrow S$ be a continuous surjective homomorphism. Let $\mu \mathrm{\vee}: \bar{\Omega}_{n} \vee \rightarrow \overline{\langle X\rangle} \subseteq$ $\bar{\Omega}_{m} \vee$ be an embedding, with $X \subseteq E\left(\bar{\Omega}_{m} \mathrm{~V}\right)$. We consider the following diagram:

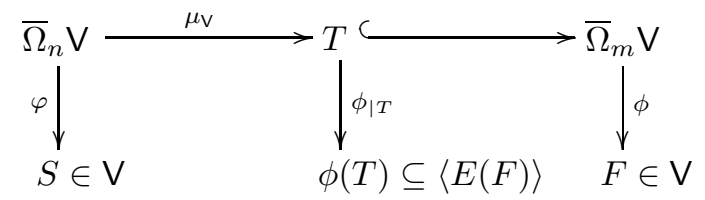

where $T$ is the image of $\mu \mathrm{v}$.

We claim that there is a family of clopen subsets $\left(U_{s}\right)_{s \in S}$ of $\bar{\Omega}_{m} \mathrm{~V}$, pairwise disjoint, such that $U_{s} \cap T=\mu_{\mathrm{V}}\left(\varphi^{-1}(s)\right)$. We proceed to prove the claim. For each $s \in S$, let $A_{s}=\mu_{\mathrm{V}}\left(\varphi^{-1}(s)\right)$ and $A_{s}^{\prime}=T \backslash A_{s}$. Since $\{s\}$ is a clopen subset of $S$, then $\varphi^{-1}(s)$ is a clopen subset of $\bar{\Omega}_{n} \vee$ and $\mu \mathrm{V}\left(\varphi^{-1}(s)\right)$ is a clopen subset of $T$. Since $A_{s}$ and $A_{s}^{\prime}$ are closed sets of the closed subspace $T$ of $\bar{\Omega}_{m} \mathrm{~V}, A_{s}$ and $A_{s}^{\prime}$ are closed subsets of $\bar{\Omega}_{m} \mathrm{~V}$. Moreover, since $\bar{\Omega}_{m} \mathrm{~V}$ is compact and 0-dimensional, then $A_{s}$ and $A_{s}^{\prime}$ are separated by two disjoint clopen sets $V_{s}$ and $V_{s}^{\prime}$, respectively. We choose an arbitrary ordering for the elements of $S: s_{1}, \ldots, s_{|S|}$. Let $U_{s_{1}}=V_{s_{1}}$ and recursively, for $i=2, \ldots,|S|$, let $U_{s_{i}}=V_{s_{i}} \backslash\left(\bigcup_{j<i} U_{s_{j}}\right)$. Note that, for every $i, U_{s_{i}}$ is also a clopen subset of $\bar{\Omega}_{m} \vee$ and $\left(U_{s}\right)_{s \in S}$ is a family satisfying the claim.

Since $\bar{\Omega}_{m} \vee$ is a pro- $\vee$ semigroup and, for all $s \in S, U_{s}$ is a clopen subset, there exists a continuous homomorphism $\phi_{s}: \bar{\Omega}_{m} \mathrm{~V} \rightarrow F_{s}$ with $F_{s} \in \mathrm{V}$ such that $U_{s}=\phi_{s}^{-1}\left(\phi_{s}\left(U_{s}\right)\right)$ (cf. [1, 2]). Let $\phi: \bar{\Omega}_{m} \vee \rightarrow F=\prod_{s} F_{s}$ be the continuous homomorphism such that $\phi=\left(\phi_{s}\right)_{s \in S}$. Then $U_{s}=\phi^{-1}\left(\phi\left(U_{s}\right)\right)$ for all $s \in S$. We 
consider the diagram

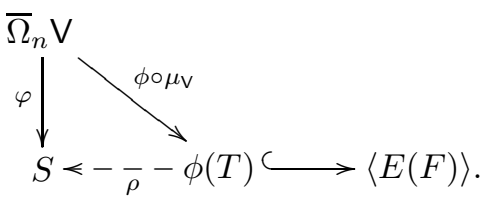

We show that $S$ is a homomorphic image of $\phi(T)$, more precisely that, there exists $\rho: \phi(T) \rightarrow S$ such that the diagram commutes. It suffices to show that, for $w, z \in$ $\bar{\Omega}_{n} \mathrm{~V}$, if $(\phi \circ \mu \mathrm{V})(w)=(\phi \circ \mu \mathrm{V})(z)$, then $\varphi(w)=\varphi(z)$. Let $s_{1}=\varphi(w), s_{2}=\varphi(z)$ and suppose that $s_{1} \neq s_{2}$. Since $U_{s_{1}} \cap U_{s_{2}}=\emptyset$, we have that $\phi\left(U_{s_{1}}\right) \cap \phi\left(U_{s_{2}}\right)=\emptyset$. Now, $\mu_{\mathrm{V}}(w) \in \mu_{\mathrm{V}}\left(\varphi^{-1}\left(s_{1}\right)\right)=U_{s_{1}} \cap T$ and, therefore, $\phi\left(\mu_{\mathrm{V}}(w)\right) \in \phi\left(U_{s_{1}} \cap T\right)$. Similarly, we obtain $\phi\left(\mu_{\mathrm{V}}(z)\right) \in \phi\left(U_{s_{2}} \cap T\right)$. It follows that $\phi\left(\mu_{\mathrm{V}}(w)\right) \neq \phi\left(\mu_{\mathrm{V}}(z)\right)$.

We conclude that $S$ divides $\langle E(F)\rangle$. Since $F \in \mathrm{V}$, it follows that $\langle E(F)\rangle \in \mathrm{V}_{\mathrm{E}}$ and, therefore, $S \in \mathrm{V}_{\mathrm{E}}$. This shows that $\mathrm{V} \subseteq \mathrm{V}_{\mathrm{E}}$, while the reverse inclusion is always verified.

From Theorem 5.1 to conclude that $\mathrm{V}_{\mathrm{E}}=\mathrm{V}$, it suffices to exhibit an embedding $\mu_{\mathrm{\vee}}: \bar{\Omega}_{n} \mathrm{\vee} \rightarrow \overline{\langle X\rangle}$ with $X \subseteq E\left(\bar{\Omega}_{m} \mathrm{\vee}\right)$, for every integer $n$. We do not know if, conversely, such an embedding always exists in case $V_{E}=V$.

For $\vee \in\{R, L, J, D A\}$, we consider the unique continuous homomorphism $\mu \vee$ such that

$$
\begin{aligned}
\mu_{\mathrm{V}}: \bar{\Omega}_{n} \mathrm{~V} & \rightarrow \bar{\Omega}_{n+1} \mathrm{\vee} \\
x_{i} & \mapsto x_{i}^{\omega} y^{\omega},
\end{aligned}
$$

where $y$ is a new variable and we prove that $\mu_{\mathrm{V}}$ is an embedding. In each case, we depend heavily on a suitable representation of the profinite semigroup $\bar{\Omega}_{n} \mathrm{~V}$.

Let us start with the pseudovariety R. We use the representation of implicit operations over $\mathrm{R}$ by means of labeled ordinals due to Almeida and Weil [3]. We briefly recall it. Let $A$ be an alphabet with $|A|=n$ and let $\mathbf{r L O}(A)$ be the set of reduced $A$-labeled ordinals. Recall that an $A$-labeled ordinal is a pair $(\alpha, l)$, where $\alpha$ is an ordinal and $l: \alpha \rightarrow A$ is a labeling function. The content of $(\alpha, l), c(\alpha, l)$, is the range of $l$. The cumulative content of a limit ordinal $\beta \leq \alpha, \overleftarrow{c}(\beta)$, is the set of all letters $a \in A$ such there exists a sequence $\left(\gamma_{k}\right)_{k}$ of ordinals with $\cup_{k} \gamma_{k}=\beta$, $\gamma_{k}<\beta$ and $l\left(\gamma_{k}\right)=a$ for all $k$. An $A$-labeled ordinal $(\alpha, l)$ is said to be reduced if $l(\beta) \notin \overleftarrow{c}(\beta)$ for each limit ordinal $\beta<\alpha$

Let $(\alpha, l) \in \mathbf{r L O}(A)$. For each $a \in A$, let $\gamma_{a}$ be the smallest ordinal such that $\gamma_{a}<\alpha$ and $l\left(\gamma_{a}\right)=a$ (i.e., $\gamma_{a}$ is the position of the first occurrence of $\left.a\right)$. We set $\gamma_{a}=0$ if $l(\gamma) \neq a$, for all $\gamma<\alpha$. Let $\alpha_{1}=\max \left\{\gamma_{a} \mid a \in A\right\}$ (i.e., the first occurrence of the last appearing letter) and let $\beta_{1}$ be such that $\alpha=\alpha_{1}+1+\beta_{1}$, with $\left(\alpha_{1}, l_{1}\right),\left(\beta_{1}, m_{1}\right) \in \mathbf{r L O}(A), l_{1}=l_{\mid \alpha_{1}}$ and $m_{1}(\gamma)=l\left(\alpha_{1}+1+\gamma\right)$ where $\gamma<\beta_{1}$. We call the equality $\alpha=\alpha_{1}+1+\beta_{1}$ the left basic partition of $(\alpha, l)$. We iterate this process while $\beta_{i} \neq 0$. Let $\beta_{0}=\alpha, m_{0}=l$ and $\beta_{i}=\alpha_{i+1}+1+\beta_{i+1}$ with $\alpha_{i+1}$ and $\beta_{i+1}$ constructed in the same way. While $\beta_{i} \neq 0$, we obtain ordinals $\left(\alpha_{i}, l_{i}\right)$ and $\left(\beta_{i}, m_{i}\right)$, with $i \geq 1$, where $l_{i+1}=m_{i_{\mid \alpha_{i+1}}}$ and $m_{i+1}(\gamma)=m_{i}\left(\alpha_{i+1}+1+\gamma\right)$ where $\gamma<\beta_{i+1}$. Almeida and Weil showed that $\alpha=\sum_{i \geq 1}\left(\alpha_{i}+1\right)$ and they define the product in $\mathbf{r L O}(A)$ that follows. Let $(\alpha, l),(\beta, m) \in \mathbf{r L O}(A)$. If $\alpha$ is not a limit ordinal, then

$$
(\alpha, l)(\beta, m)=\left(\alpha+\beta, l^{\prime}\right)
$$


where $l^{\prime}(\gamma)=l(\gamma)$ if $\gamma<\alpha$ and $l^{\prime}(\alpha+\gamma)=m(\gamma)$ if $\gamma<\beta$. If $\alpha$ is a limit ordinal, then we write $\beta=\beta_{1}+\beta_{2}$ where $\beta_{1}$ is the smallest ordinal such that $m\left(\beta_{1}\right) \notin \overleftarrow{c}(\alpha)$. The product is given by

$$
(\alpha, l)(\beta, m)=\left(\alpha+\beta_{2}, l^{\prime}\right)
$$

where $l^{\prime}(\gamma)=l(\gamma)$ if $\gamma<\alpha$ and $l^{\prime}(\alpha+\gamma)=m\left(\beta_{1}+\gamma\right)$ if $\gamma<\beta_{2}$. Almeida and Weil proved that $\mathbf{r L O}(A)$ equipped with this operation is isomorphic to $\bar{\Omega}_{n} \mathrm{R}$.

Proposition 5.2. The function $\mu_{\mathrm{R}}: \bar{\Omega}_{n} \mathrm{R} \rightarrow \bar{\Omega}_{n+1} \mathrm{R}$ is an embedding.

Proof. We denote by $\psi_{A}: \bar{\Omega}_{n} \mathrm{R} \rightarrow \mathbf{r L O}(A)$ the isomorphism defined by Almeida and Weil [3], where $|A|=n$. Let $B=\{a, b: a \in A\}$ with $b \notin A$. We consider the following diagram

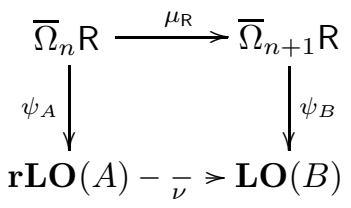

with $\nu$ defined as follows:

$$
\begin{aligned}
\nu: \mathbf{r L O}(A) & \rightarrow \mathbf{L O}(B) \\
(\alpha, l) & \mapsto\left((\omega+\omega) \alpha, l^{\prime}\right)
\end{aligned}
$$

where $\mathbf{L O}(B)$ is the set of $B$-labeled ordinals and

$$
\begin{aligned}
l^{\prime}:(\omega+\omega) \alpha & \rightarrow B \\
\beta & \mapsto \begin{cases}l(\gamma) & \text { if } \beta=(\omega+\omega) \gamma+k \text { with } \gamma<\alpha, k \in \omega \\
b & \text { if } \beta=(\omega+\omega) \gamma+\omega+k \text { with } \gamma<\alpha, k \in \omega .\end{cases}
\end{aligned}
$$

We prove that the diagram commutes, i.e., that $\nu=\psi_{B} \circ \mu_{\mathrm{R}} \circ \psi_{A}^{-1}$. Let $(\alpha=$ $\left.\sum_{i \geq 1}\left(\alpha_{i}+1\right), l\right) \in \mathbf{r L O}(A)$. Since $\psi_{A}^{-1}, \psi_{B}$ and $\mu_{\mathrm{R}}$ are homomorphisms, we proceed by induction on $\left|c\left(\alpha_{i}, l_{i}\right)\right|$, which is finite and less than $|c(\alpha, l)|$, and we obtain:

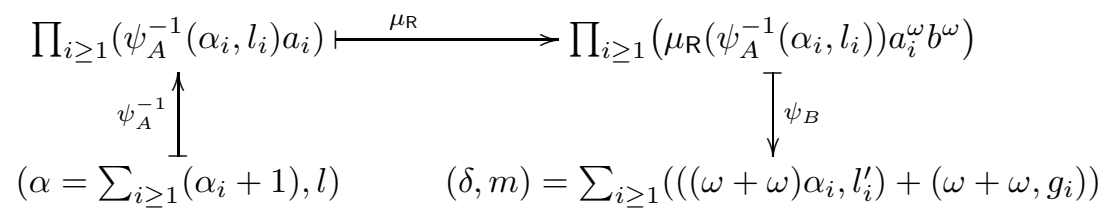

where $a_{i}=m_{i-1}\left(\alpha_{i}\right)$ and

$$
\begin{aligned}
g_{i}: \omega+\omega & \rightarrow B \\
\beta & \mapsto \begin{cases}a_{i} & \text { if } \beta<\omega \\
b & \text { if } \beta=\omega+\gamma \text { with } \gamma<\omega .\end{cases}
\end{aligned}
$$


We want to prove that $(\delta, m)=\nu(\alpha, l)=\left((\omega+\omega) \alpha, l^{\prime}\right)$. Indeed, we have

$$
\begin{aligned}
\delta & =\sum_{i \geq 1}\left((\omega+\omega) \alpha_{i}+(\omega+\omega)\right) \\
& =(\omega+\omega) \alpha_{1}+(\omega+\omega)+(\omega+\omega) \alpha_{2}+(\omega+\omega)+\cdots \\
& =(\omega+\omega)\left(\alpha_{1}+1+\alpha_{2}+1+\cdots\right) \\
& =(\omega+\omega) \sum_{i \geq 1}\left(\alpha_{i}+1\right) \\
& =(\omega+\omega) \alpha,
\end{aligned}
$$

where the third equality follows from [18, Exercise 1.41], and

$$
\begin{aligned}
m:(\omega+\omega) \alpha & B \\
\beta & \mapsto \begin{array}{ll}
l_{i}^{\prime}(\gamma) & \text { if } \beta=(\omega+\omega)\left(\sum_{j=1}^{i-1}\left(\alpha_{j}+1\right)\right)+\gamma \\
& \text { with } \gamma<(\omega+\omega) \alpha_{i} \\
g_{i}(\gamma) & \text { if } \beta=(\omega+\omega)\left(\sum_{j=1}^{i-1}\left(\alpha_{j}+1\right)\right)+(\omega+\omega) \alpha_{i}+\gamma \\
& \text { with } \gamma<(\omega+\omega),
\end{array}
\end{aligned}
$$

where we set $\sum_{j=1}^{0}\left(\alpha_{j}+1\right)=0$. In the first case, it follows that

$$
\begin{aligned}
& m(\beta)=l_{i}^{\prime}(\gamma) \\
& = \begin{cases}l_{i}^{\prime}(\delta) & \text { if } \gamma=(\omega+\omega) \delta+k, \text { with } \delta<\alpha_{i}, k \in w \\
b & \text { if } \gamma=(\omega+\omega) \delta+\omega+k, \text { with } \delta<\alpha_{i}, k \in w\end{cases} \\
& = \begin{cases}l\left(\alpha_{1}+1+\cdots+\alpha_{i-1}+1+\delta\right) & \text { if } \gamma=(\omega+\omega) \delta+k \\
b & \text { if } \gamma=(\omega+\omega) \delta+\omega+k\end{cases} \\
& = \begin{cases}l(\eta) & \text { if } \beta=(\omega+\omega) \eta+k \\
b & \text { if } \beta=(\omega+\omega) \eta+\omega+k\end{cases} \\
& =l^{\prime}(\beta)
\end{aligned}
$$

where $\eta=\sum_{j=1}^{i-1}\left(\alpha_{j}+1\right)+\delta$. In the second case, for $\eta=\sum_{j=1}^{i-1}\left(\alpha_{j}+1\right)+\alpha_{i}$, we have

$$
\begin{aligned}
m(\beta) & =g_{i}(\gamma) \\
& = \begin{cases}a_{i} & \text { if } \gamma<w \\
b & \text { if } \gamma=w+\delta \text { with } \delta<w\end{cases} \\
& = \begin{cases}l\left(\alpha_{1}+1+\cdots+\alpha_{i-1}+1+\alpha_{i}\right) & \text { if } \beta=(\omega+\omega) \eta+\delta \\
b & \text { if } \beta=(\omega+\omega) \eta+\omega+\delta\end{cases} \\
& = \begin{cases}l(\eta) & \text { if } \beta=(\omega+\omega) \eta+\delta \\
b & \text { if } \beta=(\omega+\omega) \eta+\omega+\delta\end{cases} \\
& =l^{\prime}(\beta)
\end{aligned}
$$

and, therefore, $m=l^{\prime}$. It follows that the diagram commutes and $\nu$ is a homomorphism from $\mathbf{r L O}(A)$ into $\mathbf{r L O}(B)$, where the product involved is the one defined by formulas (5.1) and (5.2). Thus, $\mu_{\mathrm{R}}$ is injective if and only if $\nu$ is injective. Let 
$(\alpha, l)$ and $(\beta, m)$ be reduced labeled ordinals such that $\nu(\alpha, l)=\nu(\beta, m)$. By $[18$, Exercise 3.41], we have

$$
(\omega+\omega) \alpha=(\omega+\omega) \beta \Longrightarrow \alpha=\beta
$$

and

$$
l^{\prime}=m^{\prime} \Longrightarrow l(\gamma)=m(\gamma) \text { for all } \gamma<\alpha \Longrightarrow l=m .
$$

Hence $(\alpha, l)=(\beta, m)$ and $\nu$ is injective.

The dual result for the pseudovariety $L$ follows by duality.

Proposition 5.3. The function $\mu_{\mathrm{L}}: \bar{\Omega}_{n} \mathrm{~L} \rightarrow \bar{\Omega}_{n+1} \mathrm{~L}$ is an embedding.

Now, we consider the pseudovariety $\mathrm{J}$ of $\mathcal{J}$-trivial semigroups. We use the representation by canonical form of implicit operations over $\mathrm{J}$ obtained by the first author [1, Section 8.2]. Consider the variety $\mathcal{V}$ of type $(2,1)$ defined by the set of identities

$$
\Sigma=\left\{(x y) z=x(y z),(x y)^{\omega}=(y x)^{\omega}=\left(x^{\omega} y^{\omega}\right)^{\omega}, x^{\omega} x=x^{\omega}=x x^{\omega},\left(x^{\omega}\right)^{\omega}=x^{\omega}\right\} .
$$

We may reduce any term in the variables $x_{1}, x_{2}, \ldots$ using the following Noetherian and confluent system of reduction rules:

(rr1): to eliminate parentheses concerning the application of the operation of multiplication;

(rr2): to substitute any subterm of the form $t^{\omega}$ by $u^{\omega}$, where $u$ is the product, in increasing order of the indices, of the variables occurring in $t$;

(rr3): to absorb in factors of the form $u^{\omega}$ any adjacent factors in which only occur variables of $u$.

A term of $\mathcal{V}$ is called a word if it does not involve the unary operation ${ }_{-}^{\omega}$, and it is called idempotent if it is of the form $t^{\omega}$, for some term $t$. The content $c(t)$ of a term $t$ is the set of variables occurring in $t$. The factorization in canonical form of a term $t$ is $t=t_{1} \cdots t_{n}$, where:

(cf1): each $t_{i}$ is a word or an idempotent;

(cf2): each idempotent $t_{i}$ is of the form $u^{\omega}$, where $u$ is a product of variables with the indices in strictly increasing order;

(cf3): given two consecutive idempotents $t_{i}$ and $t_{i+1}$, the sets $c\left(t_{i}\right)$ and $c\left(t_{i+1}\right)$ are incomparable;

(cf4): two consecutive terms $t_{i}$ and $t_{i+1}$ are not both words;

(cf5): if $t_{i}$ is a word and $t_{i+1}$ is an idempotent, then the last letter of $t_{i}$ is not in $c\left(t_{i+1}\right)$;

(cf6): if $t_{i+1}$ is a word and $t_{i}$ is an idempotent, then the first letter of $t_{i+1}$ is not in $c\left(t_{i}\right)$.

Let $F_{n} \mathcal{V}$ be the $\mathcal{V}$-free algebra on $\left\{x_{1}, \ldots, x_{n}\right\}$. The semigroup $\bar{\Omega}_{n} \mathrm{~J}$ may be seen as an algebra of type $(2,1)$, where all elements are constructed using the operations of multiplication and omega power and the variables $\left\{x_{1}, \ldots, x_{n}\right\}$. Then we have a natural surjective homomorphism

$$
\begin{aligned}
\psi: F_{n} \mathcal{V} & \rightarrow \bar{\Omega}_{n} \mathrm{~J} \\
x_{i} & \mapsto x_{i} \quad(i=1, \ldots, n),
\end{aligned}
$$

and [1, Theorem 8.2.7] establishes that $\psi$ is, in fact, an isomorphism. We are now able to prove the desired proposition. 
Proposition 5.4. The function $\mu_{\mathrm{J}}: \bar{\Omega}_{n} \mathrm{~J} \rightarrow \bar{\Omega}_{n+1} \mathrm{~J}$ is an embedding.

Proof. By the above, to show that $\mu_{\mathrm{J}}$ is injective is equivalent to establishing that

$$
\begin{aligned}
\nu: F_{n} \mathcal{V} & \rightarrow F_{n+1} \mathcal{V} \\
x_{i} & \mapsto x_{i}^{\omega} y^{\omega}
\end{aligned}
$$

is injective. Let $w, z \in F_{n} \mathcal{V}$ be such that $\nu(w)=\nu(z)$ and let $w=w_{1} \cdots w_{m}$ and $z=$ $z_{1} \cdots z_{n}$ be the respective factorizations in canonical form. We want to determine the factorizations in canonical form of $\nu(w)$ and $\nu(z)$. Let $i \in\{1, \ldots, m\}$. Suppose that $w_{i}$ is a word, i.e., $w_{i}=x_{i_{1}} \cdots x_{i_{k}}$. Then $\nu\left(w_{i}\right)=x_{i_{1}}^{\omega} y^{\omega} \cdots x_{i_{k}}^{\omega} y^{\omega}$. Note that this factorization is in canonical form, because it is a product such that two consecutive idempotents have incomparable contents. Suppose now that $w_{i}$ is an idempotent, i.e., $w_{i}=\left(x_{i_{1}} \cdots x_{i_{l}}\right)^{\omega}$ with $i_{1}<\cdots<i_{l}$. Then $\nu\left(w_{i}\right)=\left(x_{i_{1}}^{\omega} y^{\omega} \cdots x_{i_{l}}^{\omega} y^{\omega}\right)^{\omega}=$ $\left(x_{i_{1}} \cdots x_{i_{l}} y\right)^{\omega}$ applying the reduction rule $(\mathrm{rr} 2)$. Note that $i_{1}<\cdots<i_{l}<y$ (assuming that the new letter $y$ is larger than any of the others) and, therefore, the last presented factorization of $\nu\left(w_{i}\right)$ is in canonical form. Therefore, an idempotent of $F_{n} \mathcal{V}$ has as image an idempotent of $F_{n+1} \mathcal{V}$ and a word of length $k$ has as image a product of $2 k$ idempotents of $F_{n+1} \mathcal{V}$, in canonical form.

Consider now the product $w_{i} w_{j}$ with $j=i+1$. Note that, by definition of canonical form, $w_{i}$ and $w_{j}$ are not both words. Suppose that $w_{i}$ is a word and $w_{j}$ is an idempotent. Then

$$
\nu\left(w_{i} w_{j}\right)=x_{i_{1}}^{\omega} y^{\omega} \cdots x_{i_{k}}^{\omega} y^{\omega} \cdot\left(x_{j_{1}} \cdots x_{j_{l}} y\right)^{\omega}=x_{i_{1}}^{\omega} y^{\omega} \cdots x_{i_{k}}^{\omega}\left(x_{j_{1}} \cdots x_{j_{l}} y\right)^{\omega}
$$

applying the reduction rule (rr3). By hypothesis $x_{i_{k}} \notin c\left(w_{j}\right)$ and we conclude that the last factorization of $\nu\left(w_{i} w_{j}\right)$ is in canonical form. If $w_{i}$ is an idempotent and $w_{j}$ is a word, or if both $w_{i}$ and $w_{j}$ are idempotents, then we have, respectively, the following canonical forms for $w_{i} w_{j}$ :

$$
\nu\left(w_{i} w_{j}\right)=\left(x_{i_{1}} \cdots x_{i_{k}} y\right)^{\omega} x_{j_{1}}^{\omega} y^{\omega} \cdots x_{j_{l}}^{\omega} y^{\omega}
$$

and

$$
\nu\left(w_{i} w_{j}\right)=\left(x_{i_{1}} \cdots x_{i_{k}} y\right)^{\omega}\left(x_{j_{1}} \cdots x_{j_{l}} y\right)^{\omega} .
$$

Let $\nu(w)=\bar{w}_{1} \cdots \bar{w}_{m^{\prime}}$ and $\nu(z)=\bar{z}_{1} \cdots \bar{z}_{n^{\prime}}$ be the factorizations in canonical form of $\nu(w)$ and $\nu(z)$, respectively. Since $\nu(w)=\nu(z)$, by [1, Theorem 8.2.8] we have $m^{\prime}=n^{\prime}$ and $\bar{w}_{i}=\bar{z}_{i}$, for all $i$. Three cases can occur for each factor $\bar{w}_{i}$ : $\bar{w}_{i}=x_{j}^{\omega}, \bar{w}_{i}=y^{\omega}$ or $\bar{w}_{i}=\left(x_{j_{1}} \cdots x_{j_{l}} y\right)^{\omega}$, for some $j, l$. Note that the content of the idempotent in the last case has cardinal bigger than 1 , while in the other cases it is 1 . We recover $w$ as follows. In the first two cases, we substitute $\bar{w}_{i}$ by $x_{j}$ and by 1 , respectively. In the last case, we substitute $\bar{w}_{i}$ by $\left(x_{j_{1}} \cdots x_{j_{l}}\right)^{\omega}$. It is easy to see that the canonical forms of $w$ and $z$ are recovered and they are equal. It follows that $w=z$ and $\nu$ is injective.

Finally, we treat the case of DA using the representation of implicit operations over DA by means of labeled orderings obtained by Moura 11, which is similar to the case of the pseudovariety R. So, we omit most details and we refer the reader to 11 as needed. In that paper, we proved that there is a bijection between the free profinite semigroup over DA, $\bar{\Omega}_{A}$ DA, and the set of all reduced $A$-labeled $*$-linear orderings, $\mathbf{r L O} *(A)$.

Proposition 5.5. The function $\mu_{\mathrm{DA}}: \bar{\Omega}_{n} \mathrm{DA} \rightarrow \bar{\Omega}_{n+1} \mathrm{DA}$ is an embedding. 
Proof. Since $\bar{\Omega}_{A} \mathrm{DA}$ and $\mathbf{r L O}^{*}(A)$ are isomorphic, it suffices to prove that the following mapping is injective:

$$
\begin{aligned}
\nu: \mathbf{r L O}^{*}(A) & \rightarrow \mathbf{r L O}^{*}(B) \\
(o, l) & \mapsto\left(\left(\omega+\omega^{*}\right) 2 o, l^{\prime}\right) .
\end{aligned}
$$

By [1], $\nu(o, l)$ is constructed from $(o, l)$ in the following way: each position of $o$ is replaced by the ordering $\left(\omega+\omega^{*}\right) 2$ and, if this position is labeled $a \in A$, the label of each position on the resulting ordering is $a$ or $b$, depending on whether the position is in the first or second term of the form $\omega+\omega^{*}$. Thus, given two consecutive positions $p<q$ from $\nu(o, l)$, one and only one of the following cases can occur: $l^{\prime}(p)=l^{\prime}(q)=a, l^{\prime}(p)=l^{\prime}(q)=b, l^{\prime}(p)=a$ and $l^{\prime}(q)=b$, or $l^{\prime}(p)=b$ and $l^{\prime}(q)=a$, for some $a \in A$. In the first three cases, both positions are in the same interval $\left(\omega+\omega^{*}\right) 2$ of $o$, resulting from the replacement of a position of $o$ labeled $a$, for some $a \in A$. In the fourth case, the positions are in consecutive intervals corresponding to the replacement of consecutive positions of $o$. We split $\left(\omega+\omega^{*}\right) 2 o$ in intervals $I_{p}, p \in o$, of the form $\left(\omega+\omega^{*}\right) 2$ and that are maximal for the following condition: $I_{p}=I_{p, 1} \cup I_{p, 2}$ where $I_{p, 1}$ is an interval whose elements are labeled with the same letter of $A$ and $I_{p, 2}$ is an interval whose elements are labeled with $b$. It follows that $\left(\omega+\omega^{*}\right) 2 o=\bigcup_{p \in o} I_{p}$ and $l^{\prime}(\bar{p})=l(p)$, for all $\bar{p} \in I_{p, 1}$, with $I_{p}=I_{p, 1} \cup I_{p, 2}$ satisfying the above condition. Thus, we may recover $(o, l)$ from $\left(\left(\omega+\omega^{*}\right) 2 o, l^{\prime}\right)$ considering the ordering of such intervals with the labeling function that labels each interval by $a$ if the set of labels of its positions is $\{a, b\}$.

Combining Propositions [5.2, 5.3 5.4 and [5.5 with Theorem [5.1 we obtain the following result, of which only the case of DA was not already proved by the alternative approach referred to in Section 3

Corollary 5.6. The pseudovarieties $\mathrm{R}, \mathrm{L}$, J and $\mathrm{DA}$ satisfy the equation $\mathrm{V}_{\mathrm{E}}=\mathrm{V}$.

It remains an open problem whether the pseudovariety DS satisfies the equality $V_{E}=V$. This motivates the study of the free profinite semigroup over DS, for which no representation result is currently known.

\section{RANKS}

For a pseudovariety $\mathrm{V}$ we consider the following associated parameters:

- rank $\mathrm{V}$ is the least positive integer $n$ such that $\mathrm{V}$ is defined by a set of pseudoidentities on at most $n$ variables, unless there is no such $n$, in which case we let rank $\mathrm{V}=\infty$;

- for a positive integer $n, \mathrm{~V}(n)$ denotes the pseudovariety generated by all $n$-generated members of $\mathrm{V}$, that is the class of all finite continuous homomorphic images of $\bar{\Omega}_{n} \mathrm{~V}$;

- the generator rank of $\mathrm{V}$, denoted grank $\mathrm{V}$, is the least positive integer $n$ such that $\mathrm{V}=\mathrm{V}(n)$, unless there is no such $n$, in which case we set grank $\mathrm{V}=\infty$;

- the idempotent generator rank of $\mathrm{V}$, denoted idgrank $\mathrm{V}$, is the least positive integer $n$ such that $\mathrm{V}$ is generated by its members which are generated by at most $n$ idempotents, unless there is no such $n$, in which case we put idgrank $\mathrm{V}=\infty$.

The following are simple observations concerning these parameters. 
Lemma 6.1. Let $\mathrm{V}$ be a pseudovariety and $n$ a positive integer. Denote by $\Sigma_{n}$ the set of all pseudoidentities in at most $n$ variables which are valid in $\mathrm{V}$. Then the following hold:

(1) $\operatorname{rank} \mathrm{V} \leq n$ if and only if $\mathrm{V}=\llbracket \Sigma_{n} \rrbracket$;

(2) grank $\mathrm{V} \leq n$ if and only if $\mathrm{V}=\mathrm{V}(n)$;

(3) idgrank $\mathrm{V} \leq n$ implies $\mathrm{V}=\mathrm{V}_{\mathrm{E}}(n)$;

(4) $\operatorname{grank} \mathrm{V} \leq$ idgrank $\mathrm{V}$.

Lemma 6.2. Let $x_{1}, \ldots, x_{n}$ be $n$ distinct variables and consider the word $u_{i}=$ $x_{1} \cdots x_{i-1} x_{i+1} \cdots x_{n}$. Then the pseudoidentity

$$
\left(u_{1}^{\omega} \cdots u_{n}^{\omega}\right)^{\omega+1}=u_{1}^{\omega} \cdots u_{n}^{\omega}
$$

holds in $\mathrm{DS}(n-1)$ but not in $\mathrm{J}(n)$.

Proof. Ordering the variables by increasing order of the indices, the canonical form of the implicit operation over $\mathrm{J}$ determined by the left side of the pseudoidentity (6.1) is $\left(x_{1} \cdots x_{n}\right)^{\omega}$ while the right side is already in canonical form. By [1, Theorem 8.2.7] it follows that $\bar{\Omega}_{n} \mathrm{~J}$ fails (6.1), whence so does $\mathrm{J}(n)$.

Let $\varphi: \bar{\Omega}_{n} \mathrm{~S} \rightarrow \bar{\Omega}_{n-1}$ DS be any continuous homomorphism. We need to show that $\varphi$ identifies the two sides of (6.1), that is that $\varphi\left(u_{1}^{\omega} \cdots u_{n}^{\omega}\right)$ is regular. Now, by [1, Theorems 8.1.10 and 8.2.7], $\varphi\left(u_{1}^{\omega} \cdots u_{n}^{\omega}\right)$ is regular if and only if it has the same content as some $\varphi\left(u_{i}^{\omega}\right)$. Since $c\left(\varphi\left(u_{1}^{\omega} \cdots u_{n}^{\omega}\right)\right)$ contains at most $n-1$ variables, there is an index $i \in\{1, \ldots, n\}$ such that $c\left(\varphi\left(u_{1}^{\omega} \cdots u_{n}^{\omega}\right)\right)=\bigcup_{j \in\{1, \ldots, n\} \backslash\{i\}} c\left(\varphi\left(x_{j}\right)\right)$, whence $c\left(\varphi\left(u_{1}^{\omega} \cdots u_{n}^{\omega}\right)\right)=c\left(\varphi\left(u_{i}^{\omega}\right)\right)$.

The following result is an immediate application of Lemma 6.2

Proposition 6.3. Every pseudovariety in the interval [J, DS] has infinite grank.

Proof. Let $\mathrm{V}$ be a pseudovariety in the interval [J, DS] and suppose that it has finite grank $m$. Then for $n>m$ we have $\mathrm{V}=\mathrm{V}(n-1)=\mathrm{V}(n)$. This entails that $\mathrm{V}$ is contained in $\mathrm{DS}(n-1)$ and contains $\mathrm{J}(n)$, which contradicts Lemma 6.2.

In contrast, the pseudoidentity definitions of the pseudovarieties J, R, L, DA, DG, DO, DS given at the end of Section 2 show that the rank of each of them is two. Indeed, the smallest pseudovarieties defined by one-variable pseudoidentities containing the first four, respectively the last three, of these are respectively $\mathrm{A}$ and $\mathrm{S}$.

There are pseudovarieties whose generator rank is smaller than its rank. As an example, we consider the pseudovariety $\mathrm{V}=\mathrm{J}(2)$. It is obvious that $\mathrm{V}=\mathrm{V}(2)$ and so the generator rank of $\mathrm{V}$ is two (note that, for example, the semigroup $\left\langle e, f \mid e^{2}=e, f^{2}=f, f e=0\right\rangle$ is in $\left.\mathrm{J}(2) \backslash \mathrm{J}(1)\right)$. Since, by Proposition 6.3 $\mathrm{J}$ has infinite generator rank, it follows that $\mathrm{J}(2) \subsetneq \mathrm{J}$. Now, by Lemma 6.4 below, we conclude that $\mathrm{J}(2)$ has rank bigger than two.

Lemma 6.4. Let $\Sigma_{2}$ be the set of all pseudoidentities in at most two variables which are valid in $\mathrm{J}(2)$. Then $\llbracket \Sigma_{2} \rrbracket=\mathrm{J}$.

Proof. Let $u, v \in \bar{\Omega}_{2} \mathrm{~S}$ be such that the pseudoidentity $u=v$ holds in $\mathrm{J}(2)$. For every semigroup $S \in \mathrm{J}$ and every continuous homomorphism $\varphi: \bar{\Omega}_{2} S \rightarrow S$, the elements $\varphi(u)$ and $\varphi(v)$ belong to a two-generated subsemigroup of $S$, which in turn is in $\mathrm{J}(2)$. Thus we have the equality of $\varphi(u)$ and $\varphi(v)$ and so the pseudoidentity $u=v$ holds in $\mathrm{J}$. 
For the direct inclusion, it suffices to note that, if $u=v$ is a pseudoidentity in at most two variables which is valid in $\mathrm{J}$, then it is obviously valid in $\mathrm{J}(2)$, and so it belongs to $\Sigma_{2}$. Since rank $\mathrm{J}=2$, it follows that $\llbracket \Sigma_{2} \rrbracket \subseteq \mathrm{J}$.

At this point, we do not know what is the rank of the pseudovariety $J(2)$ not even if it is finite. Of course, if $\mathrm{J}(2)$ is finitely based, then it has finite rank; but we also do not know if that is the case.

For the idgrank, we can prove the following results.

Lemma 6.5. We define, recursively, two sequences of implicit operations as follows: for $n \geq 3$, we put

$$
\begin{aligned}
v_{3} & =\left(x_{1} x_{2}\right)^{\omega} x_{3} \cdot x_{1}^{2} \cdot x_{3}\left(x_{1} x_{2}\right)^{\omega} \\
w_{3} & =\left(x_{1} x_{2}\right)^{\omega} x_{3} \cdot x_{1} \cdot x_{3}\left(x_{1} x_{2}\right)^{\omega} \\
v_{n+1} & =\left(x_{1} \cdots x_{n}\right)^{\omega} x_{n+1} \cdot v_{n} \cdot x_{n+1}\left(x_{1} \cdots x_{n}\right)^{\omega} \\
w_{n+1} & =\left(x_{1} \cdots x_{n}\right)^{\omega} x_{n+1} \cdot w_{n} \cdot x_{n+1}\left(x_{1} \cdots x_{n}\right)^{\omega} .
\end{aligned}
$$

(a) If $S$ is a semigroup from DA generated by $n \geq 2$ idempotents, then $S$ satisfies the pseudoidentity $v_{n+1}=w_{n+1}$.

(b) The pseudovariety $\mathrm{J}(n)$ fails the pseudoidentity $v_{n+1}=w_{n+1}$.

Proof. (国) By hypothesis, there exists some continuous homomorphism $\pi: \bar{\Omega}_{n} \mathrm{DA} \rightarrow$ $S$ that maps the free generators $x_{i}$ to idempotents.

We proceed by induction on $n$. Given $\varphi: \bar{\Omega}_{n+1} S \rightarrow S$, we must show that $\varphi\left(v_{n+1}\right)=\varphi\left(w_{n+1}\right)$. Since $\pi$ is onto and $\bar{\Omega}_{n} \mathrm{~S}$ is free profinite, $\varphi$ factors through $\pi$, say as $\varphi=\pi \circ \psi$ for some continuous homomorphism $\psi: \bar{\Omega}_{n+1} \mathrm{~S} \rightarrow \bar{\Omega}_{n}$ DA.

At the basis of the induction, let us consider first the case $n=2$. If $\psi\left(x_{1} x_{2}\right)$ has full content, then $\varphi\left(\left(x_{1} x_{2}\right)^{\omega}\right)$ belongs to the minimum ideal of $S$. Since this ideal is a rectangular band and both $\varphi\left(v_{3}\right)$ and $\varphi\left(w_{3}\right)$ are $\mathcal{L}$ and $\mathcal{R}$-below $\varphi\left(\left(x_{1} x_{2}\right)^{\omega}\right)$, they are both equal to this idempotent. Otherwise, $\psi\left(x_{1} x_{2}\right)$ only involves one of the free generators of $\bar{\Omega}_{n}$ DA and so $\varphi\left(x_{1}\right)$ is an idempotent, in which case the equality $\varphi\left(v_{3}\right)=\varphi\left(w_{3}\right)$ is trivially verified.

For the general case $n>2$, similarly, if $\psi\left(x_{1} \cdots x_{n}\right)$ has full content, then $\varphi\left(v_{n+1}\right)=\varphi\left(w_{n+1}\right)$. Otherwise, let $T=\overline{\left\langle x_{1}, \ldots, x_{n}\right\rangle}$ so that $\varphi(T)$ is a semigroup of DA generated by at most $n-1$ idempotents. By the induction hypothesis, it satisfies the pseudoidentity $v_{n}=w_{n}$, whence $\varphi\left(v_{n}\right)=\varphi\left(w_{n}\right)$. Taking into account the definition of $v_{n+1}$ and $w_{n+1}$, we conclude that $\varphi\left(v_{n+1}\right)=\varphi\left(w_{n+1}\right)$.

(b) Let $\varphi=\bar{\Omega}_{n+1} \mathrm{~S} \rightarrow \bar{\Omega}_{n} \mathrm{~J}$ be the continuous homomorphism that fixes $x_{1}$ and sends each other $x_{i}$ to $x_{i-1}$. Consider the factorizations of $v_{n+1}$ and $w_{n+1}$ in idempotents and maximal explicit factors between them which results from the recursive definition of these implicit operations. Then a straightforward induction shows that both these factorizations and the result of applying $\varphi$ to each factor (and eliminating the repetition of $x_{1}$ within each $\omega$-power) are in canonical form. Hence $\varphi\left(v_{n+1}\right) \neq \varphi\left(w_{n+1}\right)$ by [1] Theorem 8.2.7]. Since $\bar{\Omega}_{n} \mathrm{~J}$ is residually finite, this shows that there is some member of $\mathrm{J}(n)$ that fails $v_{n+1}=w_{n+1}$.

In view of the definitions, Lemma 6.5 yields the following result.

Proposition 6.6. The inequality idgrank $\mathrm{V}(n)>n$ holds for every pseudovariety $\mathrm{V}$ in the interval [J, DA].

Combining Propositions 6.6, 5.4 5.2, 5.3 and 5.5 yields the following result. 
Corollary 6.7. The equality idgrank $\mathrm{V}(n)=n+1$ holds for every pseudovariety $\mathrm{V}$ in $\{\mathrm{J}, \mathrm{R}, \mathrm{L}, \mathrm{DA}\}$.

We finish the paper with a brief comparison of the results obtained here for the equality $\mathrm{V}=\mathrm{V}_{\mathrm{E}}$, with $\mathrm{V} \in\{\mathrm{J}, \mathrm{R}, \mathrm{L}\}$, and the results that follow from the work of Straubing [19] and Howie [9, and of Pin [15] and Laradji and Umar [10, respectively.

Straubing showed that an $n$-element $\mathcal{J}$-trivial monoid divides $\mathcal{C}_{n+1}$, and Pin proved that a finite $\mathcal{R}$-trivial monoid with cardinal $n$ embeds into $\mathcal{E}_{n}$.

On the other hand, several works deal with the ranks and idempotent ranks of various finite transformation semigroups. Recall that the rank of a finite semigroup is the minimum number of generators, and the idempotent rank of an idempotentgenerated finite semigroup is the minimum number of idempotent generators. Gomes and Howie 7] showed that the rank and idempotent rank of the subsemigroup of $\mathcal{T}_{n}$ consisting of all full transformations with range less than $n$ are both equal to $n(n-1) / 2$. The rank and idempotent rank of the subsemigroup of all contractive finite full transformations are both equal to $n+1$, as showed by Umar [20. Finally, Laradji and Umar [10] proved that the rank and idempotent rank of the subsemigroup of all contractive and order-preserving finite full transformations are both equal to $n$. We refer the reader to [21, 10] for detailed information on this topic.

Therefore, in the results quoted in Section 3 that follow from the works of these authors, the idempotent rank of the idempotent-generated semigroup is related with the cardinality of the embedded semigroup. In this section together with Section [5] we proved that any finite semigroup of J, R, L or DA with rank $n$ divides an idempotent-generated semigroup of the same type with idempotent rank at most $n+1$ (cf. Corollary 6.7). So, here the control on the number of generators is related with the number of generators of the embedded semigroup, which may be much smaller than its cardinality.

Acknowledgments. The authors are grateful to the anonymous referee for the careful reading of a preliminary version of this paper and for several helpful historical comments.

The work of both authors was supported by Fundação para a Ciência e a Tecnologia (FCT) through the Centro de Matemática da Universidade do Porto (CMUP) and also through the project PTDC/MAT/65481/2006, which is partly funded by the European Community Fund FEDER. The work of the second author was also supported by the FCT PhD Grant SFRH/BD/19720/2004.

\section{REFERENCES}

[1] J. Almeida, Finite Semigroups and Universal Algebra, World Scientific, Singapore, 1994. English translation.

[2] - Profinite semigroups and applications, in Structural Theory of Automata, Semigroups, and Universal Algebra, V. B. Kudryavtsev and I. G. Rosenberg, eds., vol. 207 of NATO Science Series II: Mathematics, Physics and Chemistry, New York, 2005, Springer, 145. Proceedings of the NATO Advanced Study Institute on Structural Theory of Automata, Semigroups and Universal Algebra, Montréal, Québec, Canada, 7-18 July 2003.

[3] J. Almeida and P. Weil, Free profinite R-trivial monoids, Int. J. Algebra and Comput. 7 (1997) 625-671.

[4] K. Auinger, T. E. Hall, N. R. Reilly, and S. Zhang, Congruences on the lattice of pseudovarieties of finite semigroups, Int. J. Algebra and Comput. 7 (1997) 433-455. 
[5] C. Benzaken and H. C. Mayr, Notion de demi-bande, demi-bandes de type deux, Semigroup Forum 10 (1975) 115-128.

[6] S. Eilenberg, Automata, Languages and Machines, vol. B, Academic Press, New York, 1976.

[7] G. Gomes and J. Howie, On the ranks of certain finite semigroups of transformations, Math. Proc. Cambridge Phil. Soc. 101 (1987) 395-403.

[8] J. M. Howie, The subsemigroup generated by the idempotents of a full transformation semigroup, J. London Math. Soc. 41 (1966) 707-716.

[9] - Products of idempotents in certain semigroups of order-preserving transformations, Proc. Edinburgh Math. Soc. 17 (1971) 223-236.

[10] A. Laradji and A. Umar, On certain finite semigroups of order-decreasing transformations I, Semigroup Forum 69 (2004) 184-200.

[11] A. Moura, Representations of the free profinite object over DA, Tech. Rep. CMUP 2009-26, Univ. of Porto, 2009. http://cmup.fc.up.pt/cmup/amoura/AMoura_DAfreeprofiniteobject.pdf to appear in Int. J. Algebra and Comput.

[12] E-local pseudovarieties, Tech. Rep. CMUP 2009-45, Univ. of Porto, 2009. http://cmup.fc.up.pt/cmup/amoura/AMoura_Elocalpv.pdf

[13] F. Pastijn, Embedding semigroups in semibands, Semigroup Forum 14 (1977) 247-263.

[14] M. Petrich, Embedding semigroups into idempotent generated ones, Monatsh. Math. 141 (2004) 315-322.

[15] J.-E. Pin, Varieties of Formal Languages, Plenum, London, 1986. English translation.

[16] N. R. Reilly and S. Zhang, Operators on the lattice of pseudovarieties of finite semigroups, Semigroup Forum 57 (1998) 208-239.

[17] J. Reiterman, The Birkhoff theorem for finite algebras, Algebra Universalis 14 (1982) 1-10.

[18] J. G. Rosenstein, Linear Orderings, Academic Press, New York, 1982.

[19] H. Straubing, On finite J-trivial monoids, Semigroup Forum 19 (1980) 107-110.

[20] A. Umar, On the semigroups of order-decreasing finite full transformations, Proc. Roy. Soc. Edinburgh Sect. A 120 (1992) 129-142.

[21] - On the ranks of certain finite semigroups of order-decreasing transformations, PortugaliæMath. 53 (1996) 23-32.

(J. Almeida) Centro de Matemática e Departamento de Matemática Pura, Faculdade de Ciências, Universidade do Porto, Rua do Campo Alegre, 687, 4169-007 Porto, PortuGAL

E-mail address: jalmeida@fc.up.pt

(A. Moura) Instituto Superior de Engenharia do Porto/LEMA and Centro de Matemática da Universidade do Porto, Rua do Campo Alegre, 687, 4169-007 Porto, Portugal

E-mail address: aim@isep.ipp.pt/amoura@fc.up.pt 